\title{
Comparison of Buyang Huanwu Granules and Naoxintong Capsules in Treatment of Stable Angina Pectoris: Rationale and Design of a Randomized, Double-blind, Multicenter Clinical Trial
}

\author{
Yu Wang \\ Zhejiang Chinese Medical University https://orcid.org/0000-0001-7894-6523 \\ Yuhan Xu \\ Zhejiang Chinese Medical University \\ Ling Zhang \\ Zhejiang Chinese Medical University \\ Shuwei Huang \\ First Affiliated Hospital of Zhejiang Chinese Medical University \\ Liping Dou \\ Second Affiliated Hospital of Zhejiang Chinese Medical University \\ Jiehong Yang \\ Zhejiang Chinese Medical University \\ Wei Fu \\ Yinchuan Cardiac-Cerebral Treatment Internet Hospital \\ Peng Zhou \\ Zhejiang Chinese Medical University \\ Haitong Wan ( $\sim$ haitongw@163.com ) \\ Zhejiang Chinese Medical University
}

Study protocol

Keywords: Stable angina pectoris, Traditional Chinese medicine, Buyang Huanwu granules, Naoxintong capsules, randomized controlled trial

Posted Date: May 10th, 2021

DOl: https://doi.org/10.21203/rs.3.rs-451005/v1

License: (c) (i) This work is licensed under a Creative Commons Attribution 4.0 International License.

Read Full License 


\section{Abstract}

Background: Stable angina pectoris (SAP) is seriously threatened the health of human life currently, and the mortality is in a continuous rising stage. The current treatment strategies mainly include pharmaceutical therapy and revascularization. In China, Buyang Huanwu granules (BYHW) and Naoxintong capsule (NXT) have been used in the treatment of SAP, but it is not clear which one is better in terms of relieving symptoms and improving quality of life. Therefore, we design a clinical trial to compare the efficacy and safety between NXT and BYHW in the treatment of SAP.

Methods: This is a randomized, double-blinded, parallel controlled, multicenter clinical trial protocol. On the basis of western medicine standardized treatment, a total of 128 SAP patients will be randomly divided into intervention group 1 (NXT group), intervention group 2 (BYHW group) and control group (placebo group) at a 2:1:1 ratio. A 2-week run-in period is required prior to randomization, and 1-week baseline period and 4-week treatment period are included in this study. The primary outcome is the efficacy rate of stable angina symptom score improvement; the secondary outcomes include the effect of electrocardiogram, Seattle Angina Questionnaire scores, and the nitroglycerin consumption.

Discussion: This study will evaluate the efficacy and safety between NXT and BYHW in the treatment of SAP. The results will provide critical evidence of the Chinese herbal medicine for SAP.

Trial registration: Chinese Clinical Trials Registry ChiCTR1800015191. Registered on 13 March 2018. http://www.chictr.org.cn/showproj.aspx?proj=25818

\section{Full Text}

This preprint is available for download as a PDF.

\section{Tables}

Table 1. Characteristics of the Investigational Product 


\section{Study drug 1: Naoxintong Capsule}

Ingredients: Astragalus mongholicus Bunge [Fabaceae; Radix Astragali], Hirudo nipponia Whitman [Hirudinidae; Whitmania pigra Whitman], Boswellia carteri Birdw., [Burseraceae; Boswellia carterii], Commiphora myrrha (T.Nees) Engl., [Burseraceae; Myrrha], Salvia miltiorrhiza Bunge [Lamiaceae; Salviae Miltiorrhizae Radix Et Rhizoma], Achyranthes bidentata Blume [Amaranthaceae; Achyranthes], Neolitsea cassia (L.) Kosterm. [Lauraceae; Cassia Twig], Morus alba L. [Moraceae; Mulberry Twig], Spatholobus suberectus Dunn [Fabaceae; Caulis Spatholobi], Buthus martensii Karsch [Buthidae; Scorpion];

- Property: capsule; the contents are brown to black brown powder; bitter in taste;

- Specification: $0.4 \mathrm{~g} /$ capsule;

$\checkmark$ Bach number: 200593.

\section{Placebo 1: Capsule placebo}

- Ingredients: corn starch, silica, caramel (liquid), 2\% NXT powder and sunset yellow;

With identical color, specification, packaging, property of contents and other features with Naoxintong Capsule;

Bach number: 200501.

\section{Study drug 2: Buyang Huanwu Granule}

- Ingredients: Astragalus mongholicus Bunge [Fabaceae; Radix Astragali], Angelica sinensis (Oliv.) Diels [Apiaceae; Angelicae Sinensis Radix], Paeonia lactiflora Pall. [Paeoniaceae; Paeoniae Radix Rubra], Lumbricus rubellus (Oligochaeta, Lumbricidae), Ligusticum chuanxiong Hort. [Apiaceae; Chuan xiong Rhizoma], Carthamus tinctorius L. [Asteraceae; Carthami Flos], Prunus persica (L.) Batsch [Rosaceae; Semen Persicae].

- Property: granule; the contents are brown to black brown granules; bitter in taste;

Specification: $5.5 \mathrm{~g} /$ bag;

Bach number: 200603.

\section{Placebo 2: Granule placebo}

Ingredients: dextrin, 1\% Ligusticum chuanxiong, bitterness SA, stevioside, lemon yellow, and chocolate brown;

With identical color, specification, packaging, property of contents and other features with Buyang Huanwu granule;

Bach number: 200601.

Table 2 Measurement items and time points for data collection. 


\begin{tabular}{|c|c|c|c|}
\hline Item & $\begin{array}{l}\begin{array}{l}\text { Run-in } \\
\text { period }\end{array} \\
(-14 \pm 1) \text { day }\end{array}$ & $\begin{array}{l}\text { Baseline } \\
\text { period } \\
(-7 \sim 0) \text { days }\end{array}$ & $\begin{array}{l}\text { Intervention } \\
\text { period } \\
(28 \pm 4) \text { days }\end{array}$ \\
\hline \multicolumn{4}{|l|}{ Basic information } \\
\hline Informed consent & $x$ & & \\
\hline Inclusion/exclusion criteria & & $x$ & \\
\hline Demographic data & $x$ & & \\
\hline Randomization & & $x$ & \\
\hline Record medical history and allergy history & $x$ & & \\
\hline Record complication and symptom & $x$ & & \\
\hline Record concomitant medication & $x$ & & \\
\hline Access to the "Doctor Tao" platform & $x$ & & \\
\hline Urine pregnancy test & $x$ & & \\
\hline \multicolumn{4}{|l|}{ Safety assessment } \\
\hline Vital signs and physical examination & $x$ & $x$ & $x$ \\
\hline Blood and urine routine examination & & $x$ & $x$ \\
\hline Liver function (ALT, AST, AP, TBIL, Y-GT) & & $x$ & $x$ \\
\hline Kidney function (SCr, BUN) & & $x$ & $x$ \\
\hline Coagulation function (PT, APTT, TT, FIB, INR) & & $x$ & $x$ \\
\hline Fasting blood glucose & & $x$ & $x$ \\
\hline \multicolumn{4}{|l|}{ Efficacy indicators } \\
\hline Stable angina symptom score & & $x$ & $x$ \\
\hline Electrocardiogram & & $x$ & $x$ \\
\hline Seattle Angina Questionnaire & & $x$ & $x$ \\
\hline Nitroglycerin consumption & $x$ & $x$ & $x$ \\
\hline \multicolumn{4}{|l|}{ Other works } \\
\hline Drug distribution & $x$ & $x$ & \\
\hline Drug recycling & & $x$ & $x$ \\
\hline
\end{tabular}


Record adverse events and combined medication

Compliance judgment $x$

$x$ $x$

$\times$

Note: ALT=alanine aminotransferase, AST=aspartate aminotransferase, AP=alkaline phosphatase, TBIL=total bilirubin, $\gamma-\mathrm{GT}=\gamma$-glutamyl transpeptidase, $\mathrm{SCr}=$ serumcreatinine, $\mathrm{BUN}=$ blood urea nitrogen, $\mathrm{PT}=$ prothrombin time, APTT=actived partial thrombolastin time, $\mathrm{TT}=$ thrombin time, $\mathrm{FIB}=\mathrm{fibrinogen}$, INR=international normalized ratio.

Figures 


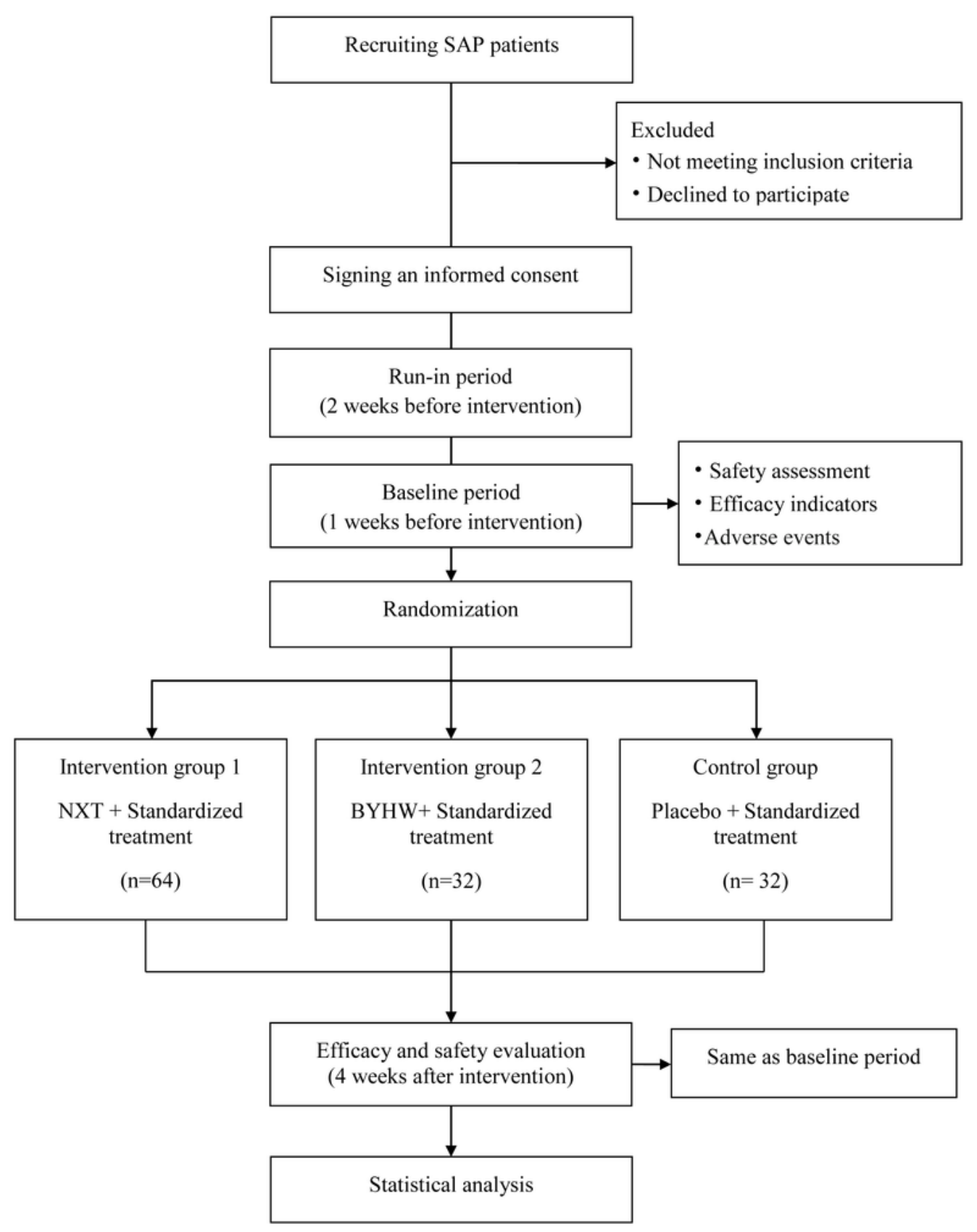

Figure 1

Study flowchart 